\title{
Model-Based Radiation-Free Assessment of Scoliosis: A Principle Validation Study
}

\author{
Susmita Roy ${ }^{1} \cdot$ Alexander T. D. Grünwald ${ }^{1} \cdot$ Renée Lampe $^{1,2}$ (-)
}

Received: 12 October 2021 / Accepted: 6 January 2022 / Published online: 18 January 2022

(c) The Author(s) 2022

\begin{abstract}
Purpose Adolescent scoliosis is one of the common pediatric spinal diseases which has a high risk of progression due to the rapid growth of the skeleton during the growing stage therefore needs regular clinical monitoring including X-rays. Because $\mathrm{X}$-rays could lead to ionizing radiation-related health problems, an ionizing radiation-free, non-invasive method is presented here to estimate the degree of scoliosis and to potentially support the medical assessment.

Methods The radiation-free body scanner provides a 3D surface scan of the torso. A basic 3D structure of the human ribcage and vertebral column was modeled and simulated with computer-aided design software and finite element method calculation. For comparison with X-rays, courses of vertebral columns derived from 3D torso images and 3D models were analyzed with respect to their apex positions and angles.

Results The methods show good results in the estimation of the apex positions of scoliosis. Strong correlations $(\mathrm{R}=0.8924)$ were found between the apex and Cobb angle from X-rays. Similar correlations $(R=0.8087)$ was obtained between the apex angles extracted from $\mathrm{X}$-rays and the combination of torso scan images with 3D model simulations. Promising agreement was obtained between the spinal trajectories extracted from X-ray and 3D torso images.

Conclusions Very strong correlations suggest that the apex angle could potentially be used for scoliosis assessment in followup examinations in complement to the Cobb angle. However, further improvements of the methods and tests on a larger number of data set are necessary before their introduction into the clinical application.
\end{abstract}

Keywords Scoliosis $\cdot$ Noninvasive $\cdot$ Ionizing radiation-free $\cdot$ Body scanner $\cdot 3 \mathrm{D}$ model of the rib cage and vertebral column · Finite element method

JEL Classification I. Health, education, and welfare $\cdot$ I1 Health $\cdot$ I10 General

\section{Introduction}

Scoliosis is a spinal deformity of at least $10^{\circ}$ curvature in the coronal plane associated with vertebrae rotation in the transverse plane [1]. Adolescent scoliosis (AS), either idiopathic or neurogenic, is one of the most prevalent types of

Renée Lampe

renee.lampe@tum.de

1 School of Medicine, Klinikum rechts der Isar, Orthopaedic Department, Research Unit of the Buhl-Strohmaier Foundation for Cerebral Palsy and Paediatric Neuroorthopaedics, Technical University of Munich, Ismaningerstr 22, 81675 Munich, Germany

2 Markus Würth Professorship, Technical University of Munich, Munich, Germany scoliosis, which has a high risk of progression and aggravation because of the rapid growth of the skeleton at the adolescent stage. Therefore, regular monitoring of AS patients and quantitative assessment of the spinal curvature are required to prevent or reduce the progression and provide proper treatment. The degree of curvature is quantified by both, measuring the Cobb angle from X-ray images [2] and clinical examinations. The Cobb angle measurement is the gold standard for scoliosis evaluation and the most reliable option for physicians to take a decision for their treatment. However, taking frequent X-rays causes a high risk of radiation-related health problems. Therefore, there is a need for supporting medical observation techniques that can reduce the number of ionizing X-ray examinations for scoliosis assessment [3]. To date, to reduce the number of $\mathrm{X}$-rays, different surface-topography based methods are the 
most commonly used alternative technique for the assessment of scoliosis [4-6]. Since lateral deviation and axial rotation of the vertebrae around the vertical body axis are strongly associated with scoliosis, 3D computational model calculations of the human rib cage and spinal column also have the potential to widen the range of assessment methods and techniques. In this regard, several mathematical and biomechanical 3D models of the spine have been developed and provide correlations between anatomical parameters and the distortions of spine, due to the changed biomechanical forces that lead to scoliosis [7-9].

The study presented here is based on the work of Roy et al. [10-12] that reported about a camera system hereinafter referred to as a body scanner, which acquires a 3D image of the human torso for the assessment of scoliosis. It is also an attempt to find an assessment method that allows one to quantify the curvature similar to the Cobb angle without radiation exposure for the patients. The present verification method is similar to Cobb's angle measurement but does not reproduce the true value of Cobb's angle; however, it correlates with Cobb's angle. In general, an X-ray image is an essential standard of care in the diagnosis of scoliosis at the first appointment of a patient with an orthopedic specialist. The present paper compares the spinal trajectories derived for a number of patients from X-ray and body scanner images and analyzes them to find one or more parameters that can be used to assess scoliosis.

\section{Materials and Methods}

\subsection{X-Ray Images}

$\mathrm{X}$-ray images from nine subjects were used to develop and evaluate the efficacy of a new method for the analysis of their 3D body torso images obtained from the body scanner.

The data set comprises patients between eleven and 30 years of age, six females and three males, and with different types of scoliosis. Table 1 shows patients' information in addition to the scoliosis assessment results evaluated by a senior orthopedic specialist from their X-ray images. With respect to the range of scoliosis, the scoliosis was classified following the characterizations: (1) thoracic, if the apex of the scoliosis curve is located between T2 and T11; (2) thoracolumbar, when located in the range T12-L1; (3) lumbar in the range L2-L5; and (4) combined, when it is a combination of thoracic and lumbar [13, 14]. Further, scoliosis was classified according to the Nash and Moe method [15].

\subsection{Scan Images and Rib Cage Model}

The 3D surface scan images of the above-mentioned patients were taken with a custom-designed body scanner, consisting of a camera system using infrared and RGB sensors, for the assessment of scoliosis [10]. The scanner system is dedicated especially for patients with cerebral palsy (CP), who are often affected by neurogenic scoliosis but also can be used for patients with idiopathic scoliosis.

The scanner is portable and can be installed in the centers for people with special needs. Patients can be scanned in both standing and sitting positions, especially many patients with CP and neurogenic scoliosis who are not able to stand for a long time. The scanning process was very fast and provided a three- dimensional surface image of the outer body shape. The system was thus non-invasive and free of ionizing radiation.

The 3D skeletal structures of male and female rib cage models were modeled [12] with computer-aided design (CAD) software [16], based on anatomical geometries and morphometric parameters derived from literature $[17,18]$. A FEBio software suite $[19,20]$, which is one of a finite element method-based [21] software tools, was then used to simulate subject-specific deformations of the vertebral column and ribcage.
Table 1 Participants' age, gender, and classification of their scoliosis evaluated from $\mathrm{X}$-ray images

\begin{tabular}{lllllll}
\hline Patient & Gender & $\begin{array}{l}\text { Age } \\
\text { Year }\end{array}$ & $\begin{array}{l}\text { Scoliosis } \\
\text { Range }\end{array}$ & Convexity & $\begin{array}{l}\text { Cobb } \\
\text { Angle in }\end{array}$ & Moe \\
\hline P1 & Female & 14 & Thoracic & Right & 34 & I \\
P2 & Female & 25 & Thoracolumbar & Right & 14 & I \\
P3 & Female & 13 & Combined & Right, left & 20,24 & I \\
P4 & Female & 11 & Thoracic & Right & 12 & I \\
P5 & Male & 30 & Thoracolumbar & Left & 12 & I \\
P6 & Male & 13 & Lumbar & Left & 14 & I \\
P7 & Male & 23 & Thoracolumbar & Right & 15 & I \\
P8 & Female & 17 & Combined & Right, left & 38,33 & II \\
P9 & Female & 15 & Thoracolumbar & Left & 42 & I \\
\hline
\end{tabular}

Moe represents the rotational degree of Moe 
Since scoliosis structure is a complicated structure, therefore the deformation was mostly simulated as anisotropic material, except few parts. The geometric and an isotropic material (where used) properties were collected from Grünwald et al. [12]. The element edge lengths of the elastic components varied from about $0.01 \sim \mathrm{mm}$ to $23 \sim \mathrm{mm}$ and thus were small in comparison to the overall geometry. Simulation on a finer meshed model showed very similar results within the principle limits.

The results were compared with the surface scan images from the body scanner system. At its best match, the rib-cage model was assumed to represent the course of the subjectspecific spine and thereby allowed to locate and mark the positions of the vertebral bodies. The trajectory of the vertebral column derived from the subject-specific distorted rib-cage model that best matches the surface scan image is therefore hereinafter referred to as the combined model.

Figure 1 depicts the afore-described derivation of the vertebral column trajectories from the body scanner and X-ray images. The surface scan image provided by the body scanner system (a.) and the simulated subject-specific deformation of the rib cage model (b.) were combined and shown in coronal (c.1) and transverse (c.2) planes. These views were then used to iteratively adapt the rib-cage model simulation to the surface scan. At its best match, the positions of vertebral bodies were marked (c.1, referred here as a combined model) and further used for analysis (f.). Ideally, on the same day of the body scan, an X-ray image of the torso was taken in the coronal plane (d.) and the positions of the vertebral bodies were marked (e.). Polynomial fits to the vertebral body positions from the combined model and X-ray then represented the vertebral column trajectories (f.) and were used for further analysis.

All the procedures related to patients' data privacy and personal interests were approved by the ethics committee of the authors' affiliated institutions.

\subsection{Analysis of Vertebral Column from X-ray Images and Scan Images}

The extracted courses of vertebral columns from two different materials (i) from the X-ray images and (ii) from the combined models were analyzed to find one or more parameter that correlates with the Cobb angle and can be used in future follow-up examinations for scoliosis assessment. To get the courses of vertebral columns, polynomials of $5^{\text {th }}$ to 9 th order were fit to the extracted scattered positions of vertebral bodies. a)

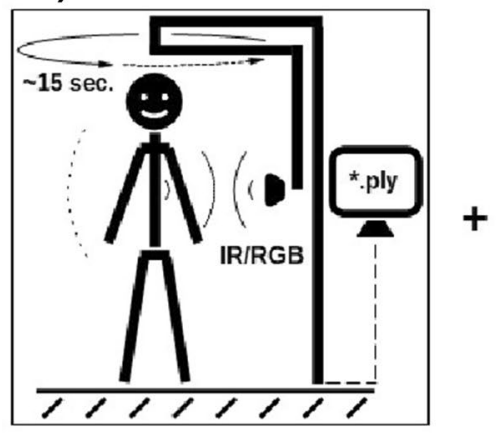

d)

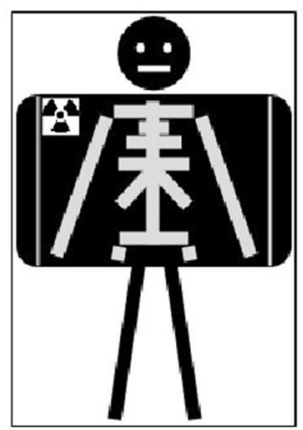

b)

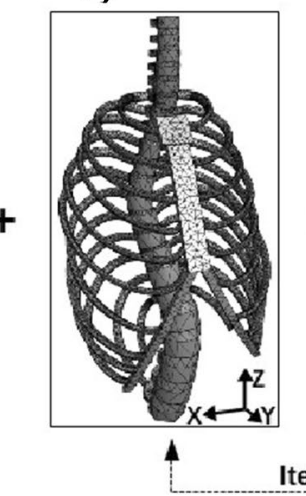

c.1)

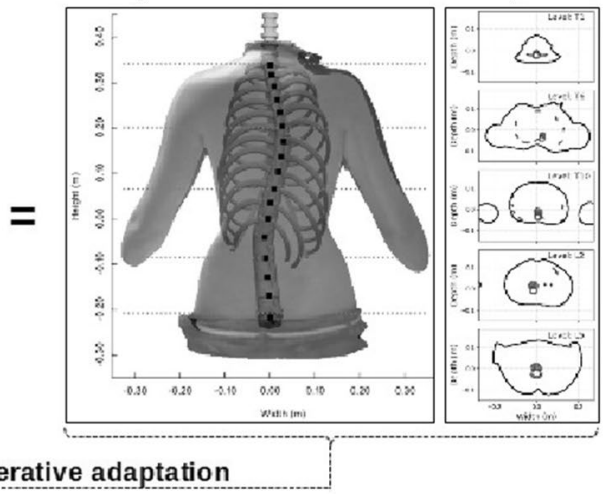

c.2)

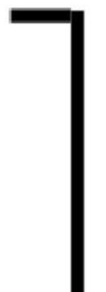

e)
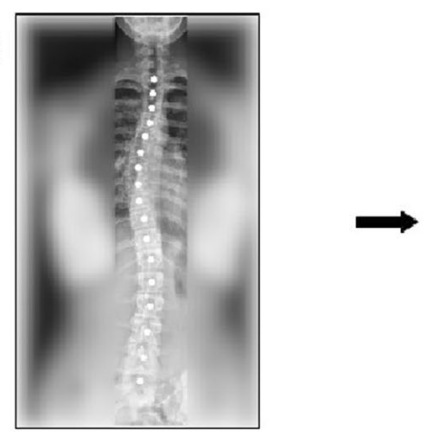

f)

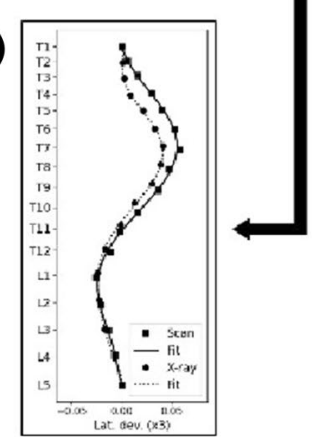

Fig. 1 Schematic diagram of the derivation of vertebral column trajectories from the combined model—surface scan image from the body scanner and subject- specific distorted rib cage simulation and X-ray images. (Further details are given in the text.) 
As an example, Fig. 2 shows 5 th order polynomial fits to the extracted scattered positions of vertebral bodies (circles) from X-ray (Fig. 2a) and combined model (Fig. 2b) from the same person. In the case of right convexity, the global maxima, and the case of left convexity the global minima of the polynomials were extracted and considered as the apex of the vertebral column. Roots of the second derivatives of the polynomials were then calculated to find potential inflection points at both sides of the apex. In addition to the apex positions (point 'A' with filled black circles), the inflection points were also marked in Fig. 2 by filled blue circles (point ' $\mathrm{B}$ ' and ' $\mathrm{C}$ '). The angle at the apex position between the lines from ' $\mathrm{A}$ ' to ' $\mathrm{B}$ ' and ' $\mathrm{A}$ ' to ' $\mathrm{C}$ ' (marked as $\theta$ in Fig. 2) was calculated and subtracted from $180^{\circ}$ to be consistent with the Cobb angle definition. This angle was defined here as the apex angle. Following this procedure, the apex angles and positions were computed from all X-ray images and combined models.

Furthermore, a quantity was calculated to evaluate the matching of the combined model with the $\mathrm{X}$-ray image. The courses of the vertebral columns derived from X-rays and model simulations were normalized to their vertical length and aligned to coincide at the top and bottom-most vertebrae $\mathrm{C} 3$, or $\mathrm{T} 1$, and $\mathrm{L} 5$, respectively. The area between the two curves was computed as a measure for the difference between the curves. The area was calculated following the equation:
$A(x, y)=\lim _{N \rightarrow \infty} \sum_{i=0}^{N-1}\left|x_{i}^{X-r a y}-x_{i}^{\bmod }\right| *\left|y_{i}-y_{i+1}\right|$

where $x^{X-r a y}$ and $x^{\text {mod }}$ are the $\mathrm{x}$-coordinates on the polynomials derived from X-ray and the combined model, and $y_{i}$ are the corresponding $\mathrm{y}$-coordinates. The latter have identical values for X-ray and the combined model. This area was computed for all the cases to see the quality of the combined model in comparison to the course of the vertebral column derived from the X-ray. Figure 3 shows overlays of the polynomial fits and the markers of the corresponding vertebrae locations representing the spinal courses.

\section{Results}

Nine X-ray images were visually analyzed by an experienced orthopedic specialist and the vertebral levels closest to the apex were determined. These levels were plotted in Fig. 4 as a function of the vertebral levels where the apex was detected by our analysis from X-ray images (Fig. 4a) and combined models (Fig. 4b). A perfect match means that the corresponding vertebral levels coincide and this would reflect in points lying over the diagonal in Fig. 4. A mismatch is indicated by the distance from the diagonal. The result shows that in most cases the vertebral level
Fig. 2 Courses of vertebral columns extracted from (a) X-ray image, (b) combined model, by fitting $5^{\text {th }}$ order polynomials to the positions of vertebral bodies denoted by circles. The filled circles mark the positions of the apex (black, point 'A') and inflections points (blue, 'B' and ' $C$ '), respectively. $\theta$ is the inner angle at the apex position between these three points. The apex angle is then defined as $(180 \circ-\theta)$
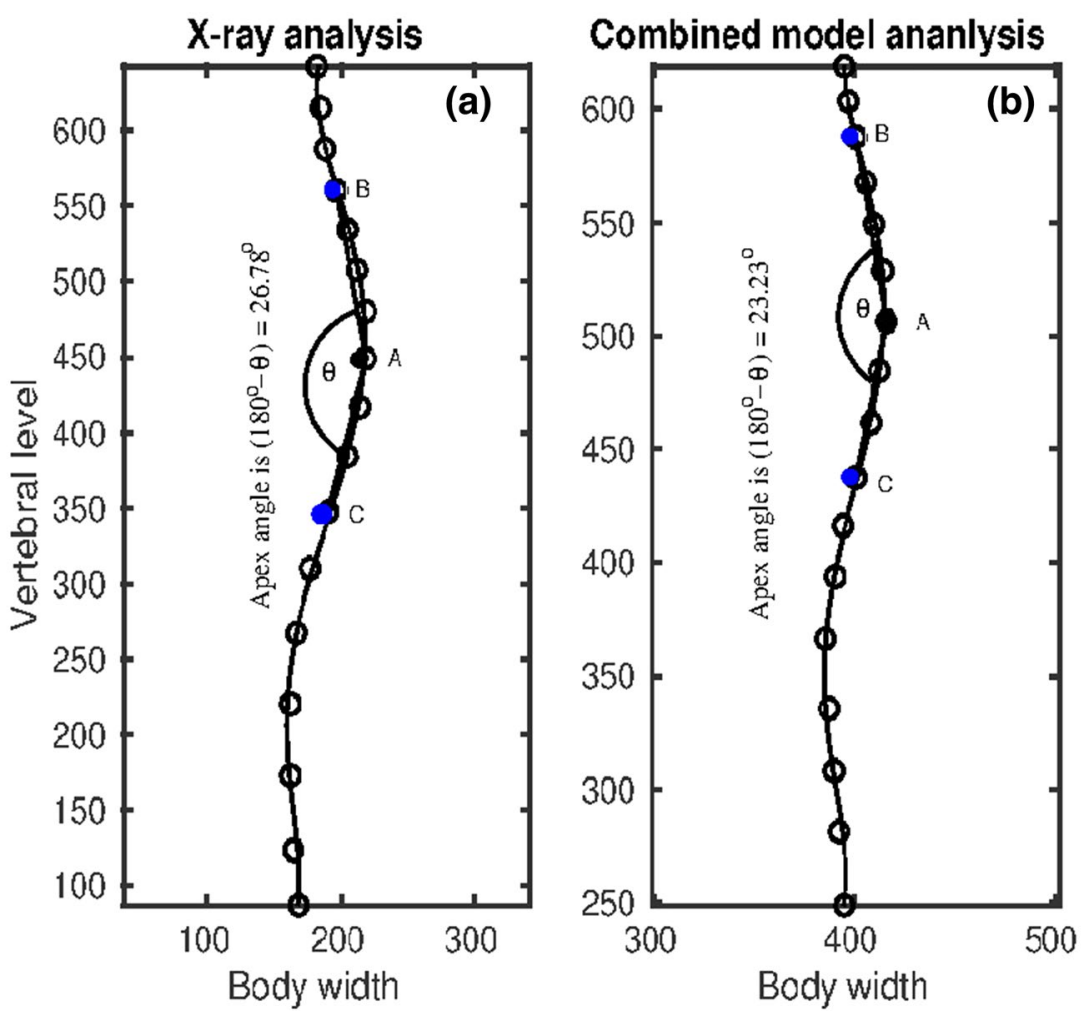

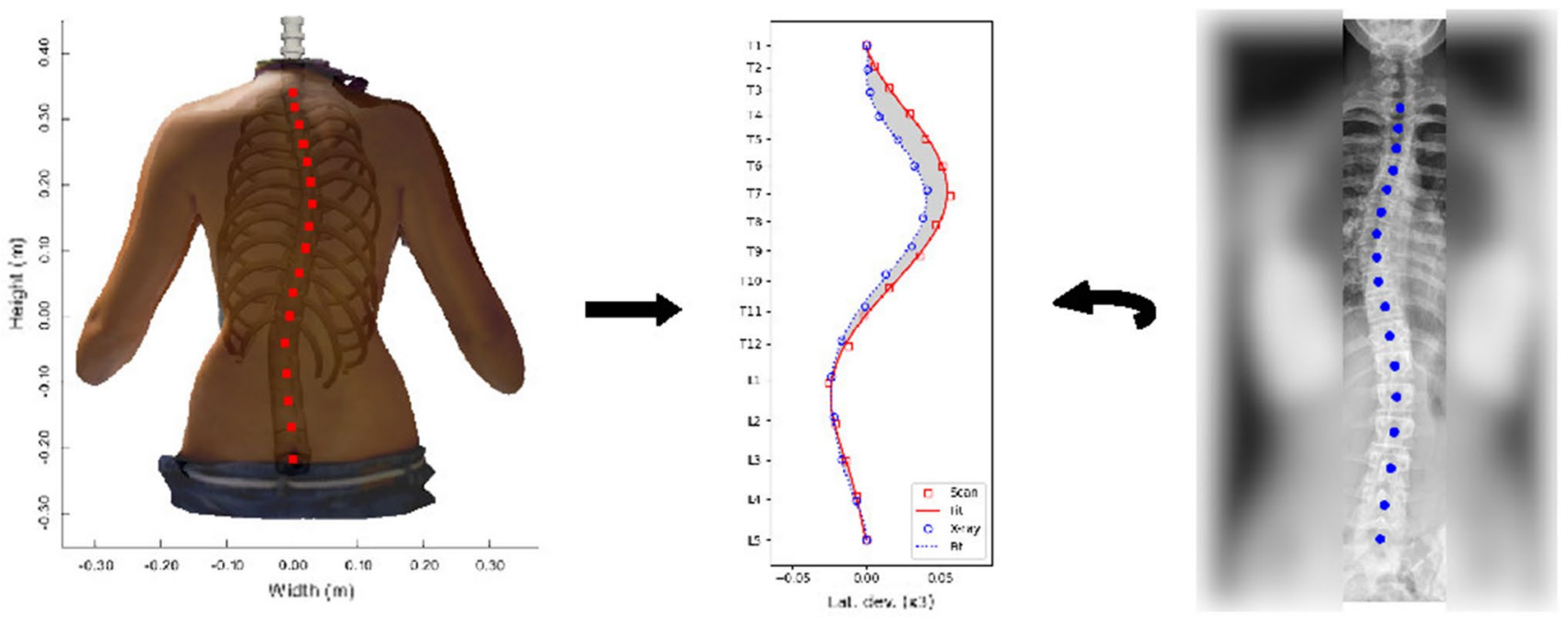

Fig. 3 Area between the polynomials (center) derived from the vertebral body positions of the combined model (left) and the X-ray (right)

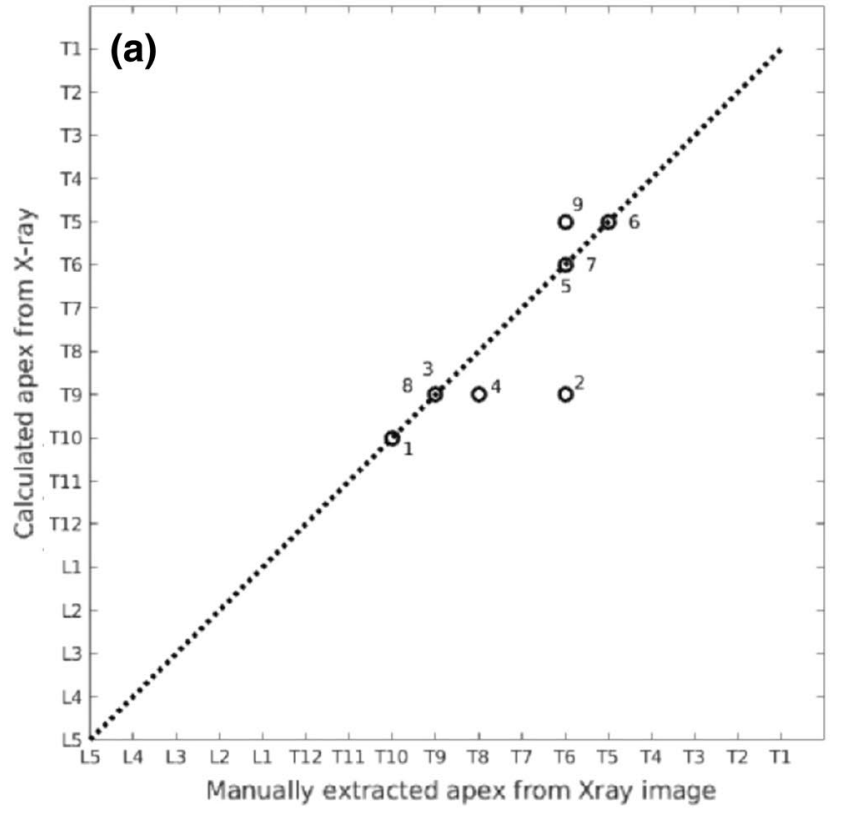

Fig. 4 Vertebral level at which apex is located as a function of the vertebral level at which apex is extracted from the analysis program. Each point represents an individual case also marked in the figure

determined by our analysis program corresponds to the vertebral level at which the apex of scoliosis occurred.

Figure 5 shows the summary of results obtained from all the X-ray images and combined models analysis. The individual bars represent the Cobb angles, the apex angles determined from the X-ray image and combined model, and the corresponding area differences. From these, a set of six Pearson's correlation coefficient values (R) were calculated following the Eq. (2). The results are presented in Table 2.

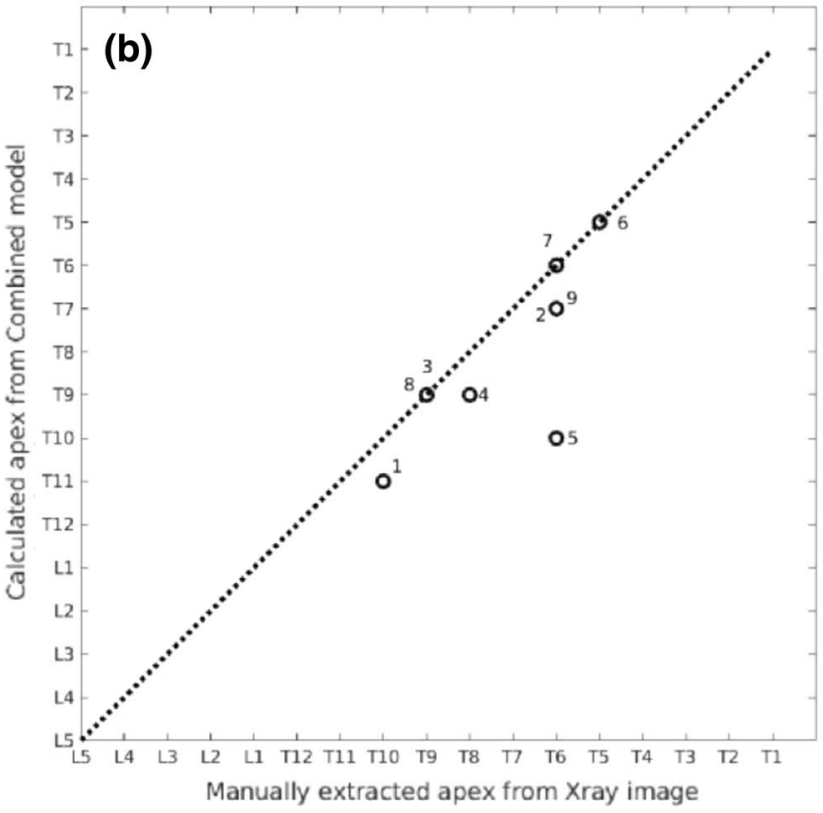

to distinguish the points where the points overlap with another case. (a) Results obtained from an X-ray image, (b) the same from combined models

$$
R=\frac{\sum\left(a_{i}-a ́ a\right)\left(b_{i}-\hat{b}\right)}{\sqrt{\sum\left(a_{i}-a\right)^{2} \sum\left(b_{i}-\hat{b}\right)^{2}}}
$$

where, $\mathrm{R}=$ correlation coefficient, $a_{i}=$ individual values of one set variable, $a=$ mean value of all $a$ variables, $b_{i}=$ individual values of another set variable, $b=$ mean value of all $b$ variables.

The $\mathrm{R}$-value ranges from -1 to 1 . $\mathrm{R}$ close to 1 , means one set of parameters increases with the increase of the second 
Fig. 5 Different parameters are plotted for each patient. Black bars indicate the Cobb angles, dark gray bars indicate the apex angles obtained from $\mathrm{X}$-ray images, gray bars indicate the apex angles obtained from the combined model and light gray indicates the area difference between the trajectories obtained from X-ray analysis and combined model analysis

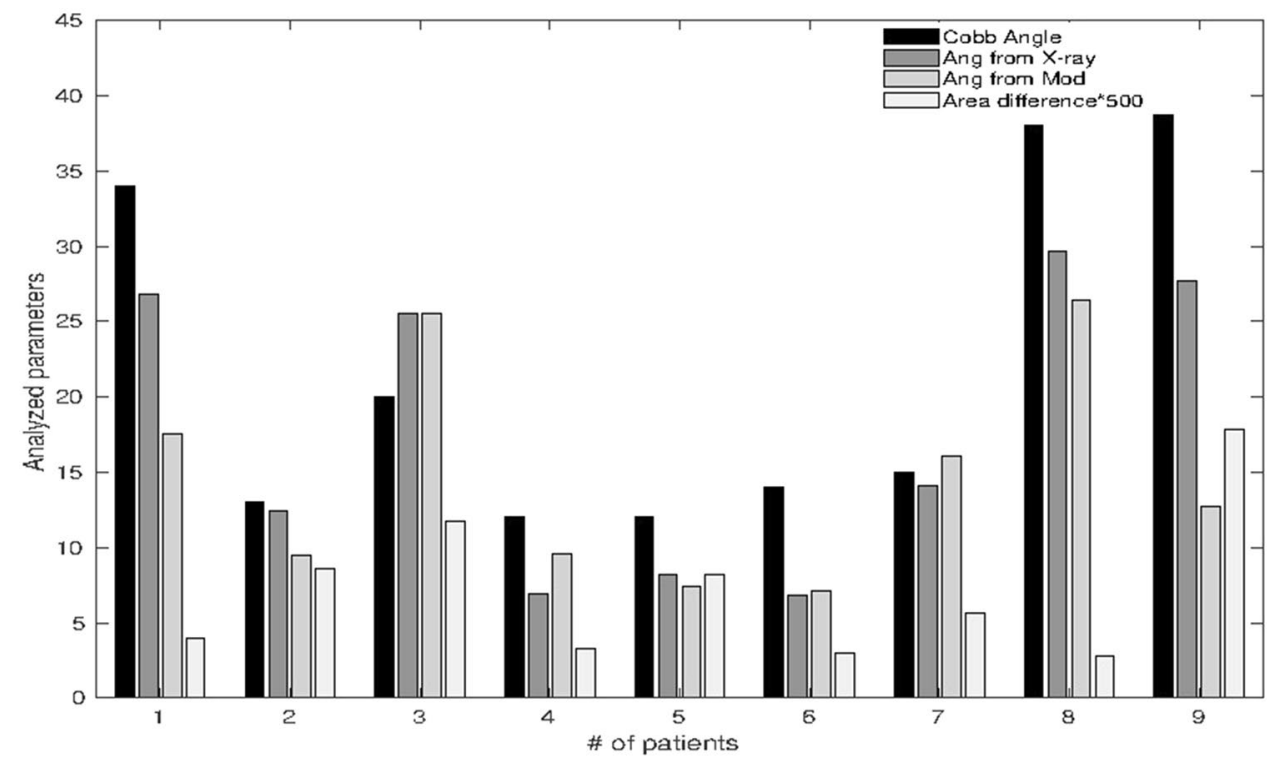

\begin{tabular}{llc}
\hline Parameters & R-value & P-value \\
\hline Cobb angle vs apex angle from the X-ray & 0.8924 & 0.0012 \\
Cobb angle vs apex angle from the combined model & 0.5328 & 0.1397 \\
Cobb angle vs area difference & 0.3182 & 0.4041 \\
Apex angle from X-ray vs apex angle from the combined model & 0.8087 & 0.0083 \\
Apex angle from X-ray vs area difference & 0.3507 & 0.3548 \\
Apex angle from combined model vs area difference & 0.0101 & 0.9794 \\
\hline
\end{tabular}

Table 2 Correlation coefficients between the analyzed parameters along with their probabilities set of parameters - a positive correlation. If R-value is close to -1 then it is a negative correlation. As a rule of thumb the absolute values of $\mathrm{R}$ can be classified into four categories: (1) weak correlation if the value lies in between 0.20 and 0.39 ; (2) moderate correlation, if it lies in between 0.4 and 0.59 ; (3) strong, when that is in between 0.6 and 0.79 ; and (4) very strong, when it is in between 0.80 and 1.00 .

The P-value is the probability that indicates when lower than the conventional 5\% $(P<0.05)$ that the correlation coefficient is statistically significant. The obtained results show that the apex angle is very strongly correlated with the Cobb angle when derived from X-ray, indicated by an R-value of $0.8924(P<0.05)$. Further, there exist a very strong correlation $(\mathrm{R}=0.8087, P<0.05)$ between the apex angles evaluated from X-ray images and combined models. The correlations between other sets of parameters are not statistically significant.

\section{Discussion}

The current work analyses the trajectories of vertebral columns extracted from (1) X-ray images and (2) a combination of body scanner images and our vertebral column and rib cage models from patients with scoliosis. The method was developed to assess adolescent scoliosis and to reduce number of X-rays in follow-ups [10, 11]. The present study included adult patients with neurogenic scoliosis due to Cerebral Palsy, where of course their growth had ended, but the patients suffered from stress-related back pain. The X-rays were taken to diagnose if degeneration alteration or other osseous dysplasia were responsible for the pain in neurogenic scoliosis. In order to develop our analysis method and to test the working principle, it was essential to include their $\mathrm{X}$-rays and body scanner images in our study.

Figure 4 showed the level of accuracy for the identification of apex positions from the analysis program and those obtained manually by an orthopedic specialist. Quite a good match between these two extraction methods supports the use of our analysis program in extracting the course of the vertebral column. The two outliers (patient P2 and patient P5) are from the cases of mild scoliosis, where a clear identification of the exact apex position was difficult by visual inspection.

A very strong correlation between the apex angles evaluated from X-ray images and the conventional Cobb angles suggests that this angle could potentially be used for scoliosis assessment in follow-up examinations complementary 
to the Cobb angle. The very strong correlation between the apex angle evaluated from $\mathrm{X}$-ray images and combined models also supports the method's potential use in follow-up examinations. However, moderate correlation between the Cobb angle and apex angle from the combined model indicates that the apex angle from the combined model is not directly comparable with the Cobb angle in the method's present state. Additionally, the method of determination and/ or the combined model potentially needs improvement. In fact, the Cobb angle measurement method is a two-dimensional measurement (i.e. on radiography) of a three-dimensional deformity of the spine. In order to be able to measure the Cobb angle, the bony curved edges of the cover and base plates of the two mostly tilted vertebral bodies around the apex, must be clearly visible in the X-ray image [22]. Therefore, even if it were possible to measure the marginal angle between the two endplates of the vertebral bodies in our 3D model that can not be exactly the same (or comparable) with the Cobb angle measured on the 2D X-ray image. Parallel lines that are drawn at the top and bottom endplates of the superior and inferior vertebral can not be drawn in 3D (i.e. the case of model) identically as in 2D (i.e. the case of X-ray image). Furthermore, natural growth-related malformations of the vertebral bodies were not considered in our present model. Here the vertebrae were designed uniformly, according to the normal anatomy. We, therefore, defined the apex angle in such a way that their way of evaluation is the same for both the cases, i.e., from X-ray and combined model. The residual mismatch between the values of either apex angle, however, might be due to the constructional differences between the real anatomy and model structure.

Neither the Cobb angle nor the apex angle is well-correlated with the area difference between the two trajectories obtained from the X-ray and combined model, nor is it statistically significant for the cases presented here. The transverse cross-sections of the 3D model provide information about the positions, axial rotations, and inclinations of the vertebral bodies (Fig. 1c.2). It is intended to include the area difference parameter as an optimization quantity for the simulation of the 3D model in the future.

With the perspective of its future application, the method presented here could come in clinical practice as follows: when a patient exhibits signs of scoliosis during his first consultation with an orthopedic specialist, in addition to the regular X-ray image a 3D surface scan of the torso will be taken with the body scanner. From either image, following the procedure presented here, apex angles will be extracted. Provided that there are very strong correlations between the Cobb angle and the apex angle from the X-ray, as well as between the apex angles from X-ray and combined model, the apex angle could be used for comparison instead of the Cobb angle. Later in follow-up examinations, in addition to the clinical examination, another non-invasive body scan will be performed. The apex angle extracted from the combined model using the latest $3 \mathrm{D}$ body scan will then be compared with the previous one and thus help to decide whether another X-ray is indicated. In case there is no significant difference in complement to the clinical examination, the orthopedic specialist may come to the conclusion that no X-ray will be required at this stage, and thus reduce the exposure to ionizing radiation for the patient. Although the present method will not eliminate the need for X-rays, especially at first time, a radiograph is required for evaluation of the morphology of the spine, the method has the potential to reduce the number of X-rays taken over the life span of a patient. Furthermore, the method is independent of the natural growth of a person and their posture during scanning. Once the method has been tested and validated on a larger number of data set, it can be applied in clinical practice.

The present method shares the technique of other modelbased image analysis methods [23-25] as well as the 3D reconstruction of the spinal curve [26] as a basis for the assessment of scoliosis. Furthermore, the advantages are associated with short computation time and full control over our model. Further developments of the model structure in more detail and improvements of the present simulation procedure are required. In future, a larger number of patients with different types of scoliosis is required to prove the method's efficacy. Follow the steps presented here an integrated software tool will be the next step to automatically perform a scoliosis assessment and monitoring.

\section{Conclusions}

An ionizing radiation-free, non-invasive method is presented to estimate the degree of scoliosis and to support the clinical assessment. The method shows good results in the estimation of the apex positions of scoliosis. Strong correlations were found between the apex angle, evaluated by our radiation-free scanning method, and Cobb angle from X-rays. Very strong correlation results suggest that the apex angle could be used for scoliosis assessment in follow-up examinations in complement to Cobb angle.

Acknowledgements The authors would like to thank the patients for their time and support in this study. Financial supports from the BuhlStrohmaier Foundation, Würth Foundation, and Klaus Tschira Foundation (Project Nr. 00.333.2017) are acknowledged. The authors would like to thank the TUM English writing section for their support to finalize the manuscript.

Funding Open Access funding enabled and organized by Projekt DEAL. 


\section{Declarations}

Conflict of interest The authors declare that they have o conflict of interest.

Ethical Approval All the procedures related to patients' data privacy and personal interests were approved by the ethics committee of the Faculty of Medicine of the Technical University of Munich, Germany (Ref 569/16 S).

Open Access This article is licensed under a Creative Commons Attribution 4.0 International License, which permits use, sharing, adaptation, distribution and reproduction in any medium or format, as long as you give appropriate credit to the original author(s) and the source, provide a link to the Creative Commons licence, and indicate if changes were made. The images or other third party material in this article are included in the article's Creative Commons licence, unless indicated otherwise in a credit line to the material. If material is not included in the article's Creative Commons licence and your intended use is not permitted by statutory regulation or exceeds the permitted use, you will need to obtain permission directly from the copyright holder. To view a copy of this licence, visit http://creativecommons.org/licenses/by/4.0/.

\section{References}

1. Staheli, L. T. (2001). Practice of pediatric orthopedics. Lippincott Williams \& Wilkins.

2. Cobb, J. (1947). Outline for the study of scoliosis. Instructional Course Lectures, 5, 261-275.

3. Presciutti, S. M., Karukanda, T., \& Lee, M. (2014). Management decisions for adolescent idiopathic scoliosis significantly affect patient radiation exposure. The Spine Journal, 14(9), 1984-1990. https://doi.org/10.1016/j.spinee.2013.11.055

4. Drerup, B. (2014). Rasterstereographic measurement of scoliotic deformity. Scoliosis, 9(1), 22. https://doi.org/10.1186/ s13013-014-0022-7

5. Adrian, G., Fiona, B., \& Paul, P. (2017). The effects of scoliosis and subsequent surgery on the shape of the torso. Scoliosis and Spinal Disorders. https://doi.org/10.1186/s13013-017-0140-0

6. Ghaneei, M., Ekyalimpa, R., Westover, L., Parent, E., \& Adeeb, S. (2019). Customized k-nearest neighbourhood analysis in the management of adolescent idiopathic scoliosis using 3D markerless asymmetry analysis. Computer Methods in Biomechanics and Biomedical Engineering, 22(7), 696-705. https://doi.org/10.1080/ 10255842.2019.1584795

7. Bassani, T., Ottardi, C., Costa, F., Brayda-Bruno, M., Wilke, H., \& Galbusera, F. (2017). Semiautomated 3d spine reconstruction from biplanar radiographic images: Prediction of intervertebral loading in scoliotic subjects. Frontiers in Bioengineering and Biotechnology, 5, 1. https://doi.org/10.3389/fbioe.2017.00001

8. Jalalian, A., Gibson, I., \& Tay, E. H. (2013). Computational biomechanical modelling of scoliotic spine: Challenges and opportunities. Spine Deformity, 1(6), 401-411. https://doi.org/10.1016/j. jspd.2013.07.009

9. Gummerson, N. W., \& Millner, P. A. (2011). (ii) scoliosis in children and teenagers. Orthopaedics and Trauma, 25(6), 403-412.

10. Roy, S., Grünwald, A. T. D., Alves-Pinto, A., Maier, R., Cremers, D., Pfeiffer, D., \& Lampe, R. (2019). A noninvasive 3D body scanner and software tool towards analysis of scoliosis. BioMed Research International, 9(2019), 4715720. https://doi.org/10. $1155 / 2019 / 4715720$

11. Roy, S., Grünwald, A. T. D., Alves-Pinto, A., \& Lampe, R. (2020). Automatic analysis method of 3D images in patients with scoliosis by quantifying asymmetry in transverse contours. Biocybernetics and Biomedical Engineering, 40, 1486-1498. https://doi.org/10. 1016/j.bbe.2020.09.001

12. Grünwald, A. T. D., Roy, S., Alves-Pinto, A., \& Lampe, R. (2021). Assessment of adolescent idiopathic scoliosis from body scanner image by finite element simulations. PLoS ONE, 16(2), e0243736. https://doi.org/10.1371/journal.pone.0243736

13. Jaremko, J., Delorme, S., Dansereau, J., Labelle, H., Ronsky, J., Poncet, P., Harder, J., Dewar, R., \& Zernicke, R. F. (2000). Use of neural networks to correlate spine and rib deformity in scoliosis. Computer Methods in Biomechanics and Biomedical Engineering, 3, 203-213. https://doi.org/10.1080/10255840008915265

14. Pope, M., Stokes, I. A., \& Moreland, M. (1984). The biomechanics of scoliosis. Critical Reviews in Biomedical Engineering, 11(3), 157-188.

15. Ng, S. Y., \& Bettany-Saltikov, J. (2017). Imaging in the diagnosis and monitoring of children with idiopathic scoliosis. The Open Orthopaedics Journal, 11, 1500-1520. https://doi.org/10.2174/ 1874325001711011500

16. Riegel, J., Mayer W., van Havre, Y., and others (2001-2021). FreeCAD (Versions 0.18 and 0.19) [Software] from: https://www. freecadweb.org/

17. Busscher, I., Ploegmakers, J. J. W., Verkerke, G. J., \& Veldhuizen, A. G. (2010). Comparative anatomical dimensions of the complete human and porcine spine. European Spine Journal, 19(7), 1104-1114. https://doi.org/10.1007/s00586-010-1326-9

18. Holcombe, S. A., Wang, S. C., \& Grotberg, J. B. (2016). Modeling female and male rib geometry with logarithmic spirals. Journal of Biomechanics, 49(13), 2995-3003. https://doi.org/10.1016/j. jbiomech.2016.07.021

19. Maas, S. A., Ellis, B. J., Ateshian, G. A., \& Weiss, J. A. (2012). FEBio: Finite elements for biomechanics. Journal of Biomechanical Engineering, 134(1), 011005. https://doi.org/10.1115/1.40056 94

20. Maas, S.A., Ellis, B.J., Ateshian, G.A., Weiss, J.A., and others (2019). FEBio (Version 2.9.0) [Software] from: https://febio.org/

21. Hui, C. L., Piao, J., Wong, M. S., \& Chen, Z. (2020). Study of textile fabric materials used in spinal braces for scoliosis. Journal of Medical and Biological Engineering, 40(3), 356-371. https:// doi.org/10.1007/s40846-020-00516-9

22. Al-Bashir, A. K., Al-Abed, M. A., Amari, H. K., Al-Rousan, F. M., Bashmaf, O. M., Abdulhay, E. W., Al Abdi, R. M., Arunkumar, N., Bapu, B. T., \& Al-Basheer, A. K. (2019). Computerbased cobb angle measurement using deflection points in adolescence idiopathic scoliosis from radiographic images. Neural Computing and Applications, 31(5), 1547-1561. https://doi.org/ 10.1007/s00521-018-3614-y

23. Forsberg, D., Lundström, C., Andersson, M., \& Knutsson, H. (2014). Model-based registration for assessment of spinal deformities in idiopathic scoliosis. Physics in Medicine \&amp; Biology, 59(2), 311-326.

24. Giannoglou, V., \& Stylianidis, E. (2016). Review of advances in cobb angle calculation and image-based modelling techniques for spinal deformities. ISPRS Annals of the Photogrammetry, Remote Sensing Spatial Information Sciences, 3(5), 129-135.

25. Dubousset, J., Charpak, G., Dorion, I., Skalli, W., Lavaste, F., Deguise, J., Kalifa, G., \& Ferey, S. (2005). A new 2d and 3d imaging approach to musculoskeletal physiology and pathology with low-dose radiation and the standing position: The eos system. Bulletin de l'Académie Nationale de Médecine, 189(2), 287-297.

26. Wu, H. D., \& Wong, M. S. (2020). Assessment of maximum spinal deformity in scoliosis: A literature review. Journal of Medical and Biological Engineering, 40(5), 621-629. https://doi.org/10. 1007/s40846-020-00558-z 